\title{
Bulk Local Operators, Conformal Descendants, and Radial Quantization
}

\author{
Zhao-Long Wang ${ }^{1,2}$ and Yi Yan ${ }^{1,2}$ \\ ${ }^{1}$ Institute of Modern Physics, Northwest University, Xian 710069, China \\ ${ }^{2}$ Shaanxi Key Laboratory for Theoretical Physics Frontiers, Xi'an 710069, China \\ Correspondence should be addressed to Zhao-Long Wang; zlwang4@gmail.com
}

Received 5 July 2017; Accepted 13 August 2017; Published 2 October 2017

Academic Editor: Zhang-Yu Nie

Copyright (c) 2017 Zhao-Long Wang and Yi Yan. This is an open access article distributed under the Creative Commons Attribution License, which permits unrestricted use, distribution, and reproduction in any medium, provided the original work is properly cited. The publication of this article was funded by SCOAP ${ }^{3}$.

We establish a construction of the bulk local operators in AdS by considering CFT at finite energy scale. Without assuming any prior knowledge about the bulk, the solution to the bulk free field equation automatically appears in the field theory arguments. In the radial quantization formalism, we find a properly regularized version of our initial construction. Possible generalizations beyond pure AdS are also discussed.

\section{Introduction}

The AdS/CFT correspondence [1-4] implies a duality between the quantum gravity in $d+1$-dimensional anti-de Sitter space and the $d$-dimensional conformal field theory which is defined on the boundary of $\mathrm{AdS}_{d+1}$. The AdS metric in the Poincaré patch is given by

$$
\mathrm{d} s^{2}=\frac{1}{z^{2}}\left(\mathrm{~d} z^{2}+\eta_{\mu \nu} \mathrm{d} x^{\mu} \mathrm{d} x^{\nu}\right)
$$

where the boundary is the $d$-dimensional flat space at $z=$ 0 . The relations between the boundary data of AdS and the CFT quantities have been well established in [4] by the field-operator correspondence. That is, the correlators of a conformal primary operator $\mathcal{O}(x)$ in the CFT are reproduced by the asymptotical data of a bulk field $\phi$ near the boundary. However, the explicit CFT construction of the bulk local degree of freedoms $\phi(x, z)$ inside the AdS space is not well understood yet. The earlier attempts [5-7] suggested to reconstruct $\phi(x, z)$ by propagating the bulk modes from the bulk to the boundary, and then [8-10] showed that it is equivalent to the smearing operator construction. In this letter, we suggest a different construction based on almost purely CFT arguments. In Section 2.1, we establish the construction by considering CFT at finite energy scale. The possible divergence and the prescription of regulator are discussed in Section 2.2. Then in Section 3, we find that our construction can get improved in the radial quantization formalism. We summarize our main results in Section 4, and a possible way of generalizing the construction beyond pure AdS is also proposed there.

\section{CFT Construction of Bulk Local Operators}

2.1. Renormalized Primary at Finite Energy Scale. It has been pointed out qualitatively $[1-4,11]$ that the bulk radial direction $z$ is related to the energy scale in the dual field theory. In order to reconstruct $\phi(x, z)$, the first candidate is to consider in CFT the renormalized primary operator $\mathcal{O}(x, \mu)$ which is defined at a finite energy scale $\mu$. On the other hand, the behaviors of a primary operator under the conformal transformation have already been encoded in its conformal family. Thus it is natural to expect, at least in the leading order, that the renormalization of the primary operator at a finite energy scale will lead to a mixing between the primary operator and its descendants

$$
\mathcal{O}(x, \mu)=Z(\mu, \partial) \mathcal{O}(x) .
$$

For simplicity, we will only consider the scalar operator from now on. It is also natural to require that the renormalized 
primary operator recovers the Lorentz properties and the scaling dimension of the original primary. Then we find it can only be in the following form:

$$
\mathcal{O}(x, \mu)=Z\left(\mu^{-2} \square\right) \mathcal{O}(x) .
$$

Is it possible to fix the explicit form of $Z\left(\mu^{-2} \square\right)$ by imposing certain renormalization condition? The idea is to give the word "primary" a renormalized meaning. In the usual CFT language, the definition of a primary operator is equivalent to requiring that it transforms as a tensor under conformal transformations. We also notice that the renormalization scale $\mu$ will transform nontrivially under conformal transformations. Thus a direct guess is that the proper renormalization condition should be the following. The renormalized primary transforms as a tensor under the generalized conformal transformations including the energy scale.

To address the generalized conformal transformations including the energy scale, let us firstly review the realization of conformal algebra on the $x$-space. Acting on the coordinates $x^{\mu}$, the conformal generator can be expressed as follows:

$$
\begin{aligned}
P_{\mu}^{\circ} & =-i \partial_{\mu}, \\
M_{\mu \nu} \circ & =-i\left(x_{\mu} \partial_{\nu}-x_{\nu} \partial_{\mu}\right), \\
D^{\circ} & =-i x^{\mu} \partial_{\mu}, \\
K_{\mu^{\circ}} & =-i\left(x^{2} \partial_{\mu}-2 x_{\mu} x^{\nu} \partial_{\nu}\right) .
\end{aligned}
$$

It implies the standard conformal algebra

$$
\begin{aligned}
& {\left[P_{\mu}, P_{\nu}\right]=0} \\
& {\left[M_{\mu \nu}, M_{\rho \sigma}\right]} \\
& \quad=i\left(\eta_{\mu \rho} M_{\nu \sigma}+\eta_{\nu \sigma} M_{\mu \rho}-\eta_{\nu \rho} M_{\mu \sigma}-\eta_{\mu \sigma} M_{\nu \rho}\right) \\
& \quad\left[M_{\mu \nu}, P_{\rho}\right]=i\left(\eta_{\mu \rho} P_{\nu}-\eta_{\nu \rho} P_{\mu}\right) \\
& {\left[M_{\mu \nu}, K_{\rho}\right]=i\left(\eta_{\mu \rho} K_{\nu}-\eta_{\nu \rho} K_{\mu}\right)} \\
& {\left[D, M_{\mu \nu}\right]=0} \\
& {\left[D, K_{\mu}\right]=-i K_{\mu},} \\
& {\left[D, P_{\mu}\right]=i P_{\mu},} \\
& {\left[K_{\mu}, K_{\nu}\right]=0} \\
& {\left[K_{\mu}, P_{\nu}\right]=-2 i M_{\mu \nu}-2 i \eta_{\mu \nu} D .}
\end{aligned}
$$

To include the energy scale (for different approaches of introducing the finite energy scale, see $[12,13]$.), a straightforward way is to add $\partial / \partial_{\mu}$ as well as $\mu$-dependent coefficients into the realization (4). For latter convenience, we define $z \equiv$ $1 / \mu$ and equivalently consider $z$ instead. From the fact that the energy scale is Poincaré invariant, we conclude that the forms of $P_{\mu}$ and $M_{\mu \nu}$ remain intact. The scaling dimension of energy scale is obviously 1 ; thus we can easily write down the following generalized form of dilatation $D$ :

$$
D \circ=-i\left(z \partial_{z}+x^{\mu} \partial_{\mu}\right)
$$

For the special conformal generator, the strategy is to take its most general ansatz

$$
K_{\mu}{ }^{\circ}=-i\left[x^{2} \delta_{\mu}^{\nu}-2 x_{\mu} x^{\nu}+f_{\mu}^{\nu}(z, x)\right] \partial_{\nu}-i g_{\mu}(z, x) \partial_{z}
$$

and then try to find the explicit form which satisfies the conformal algebra.

From $\left[K_{\mu}, P_{\nu}\right]=-2 i M_{\mu \nu}-2 i \eta_{\mu \nu} D$, we get

$$
2 \eta_{\mu \nu} z \partial_{z}=-\partial_{\nu} f_{\mu}^{\rho}(z, x) \partial_{\rho}-\partial_{\nu} g_{\mu}(z, x) \partial_{z}
$$

It implies that

$$
\begin{gathered}
f_{\mu}^{\nu}(z, x)=\delta_{\mu}^{\nu} f(z), \\
g_{\mu}(z, x)=-2 x_{\mu} z .
\end{gathered}
$$

From $\left[D, K_{\mu}\right]=-i K_{\mu}$, we further get

$$
f(z)=\alpha z^{2}
$$

where $\alpha$ is an arbitrary constant. Finally, we can check that the above results satisfy $\left[K_{\mu}, K_{\nu}\right]=0$. In conclusion, we have

$$
\begin{aligned}
P_{\mu} \circ & =-i \partial_{\mu}, \\
M_{\mu \nu} \circ & =-i\left(x_{\mu} \partial_{\nu}-x_{\nu} \partial_{\mu}\right), \\
D \circ & =-i\left(z \partial_{z}+x^{\mu} \partial_{\mu}\right), \\
K_{\mu} \circ & =-i\left[\left(x^{2}+\alpha z^{2}\right) \partial_{\mu}-2 x_{\mu} x^{\rho} \partial_{\rho}-2 x_{\mu} z \partial_{z}\right] .
\end{aligned}
$$

In fact, this is exactly the isometry generator of the AdS space when $\alpha>0$ and it suggests to identify $\sqrt{\alpha} z$ here with the standard AdS radial coordinate. We also notice that it corresponds to $\mathrm{dS}_{d+1}$ when $\alpha$ is negative, but we will only concentrate on the AdS case in this paper.

Given the generalized conformal transformation including the energy scale (11), we can try to decide the form of $Z\left(z^{2} \square\right)$ by our renormalization condition on primary. For a scalar in the $\{x, z\}$ space, we can expand it by powers of $z$

$$
\Phi(z, x)=z^{\Delta} \sum_{n=0}^{\infty} z^{n} \Phi_{n}(x)
$$

The scalar transformation rule

$$
\widetilde{\Phi}(\widetilde{z}, \tilde{x})=\Phi(z, x)
$$


implies that the terms appearing in the power expansion should transform as follows:

$$
\begin{aligned}
& {\left[P_{\mu}, \Phi_{n}(x)\right]=i \partial_{\mu} \Phi_{n}(x),} \\
& {\left[M_{\mu \nu}, \Phi_{n}(x)\right]=i\left(x_{\mu} \partial_{\nu}-x_{\nu} \partial_{\mu}\right) \Phi_{n}(x)} \\
& {\left[D, \Phi_{n}(x)\right]=i\left(\Delta+n+x^{\mu} \partial_{\mu}\right) \Phi_{n}(x),} \\
& {\left[K_{\mu}, \Phi_{0}(x)\right]=i\left[x^{2} \partial_{\mu}-2 x_{\mu} x^{\nu} \partial_{\nu}-2 x_{\mu} \Delta\right] \Phi_{0}(x),} \\
& {\left[K_{\mu}, \Phi_{1}(x)\right]} \\
& \quad=i\left[x^{2} \partial_{\mu}-2 x_{\mu} x^{\nu} \partial_{\nu}-2 x_{\mu}(\Delta+1)\right] \Phi_{1}(x), \\
& {\left[K_{\mu}, \Phi_{n}(x)\right]} \\
& \quad=i \alpha \partial_{\mu} \Phi_{n-2}(x) \\
& \quad+i\left[x^{2} \partial_{\mu}-2 x_{\mu} x^{\nu} \partial_{\nu}-2 x_{\mu}(\Delta+n)\right] \Phi_{n}(x)
\end{aligned}
$$$$
(n>1) \text {. }
$$

Now the task is to construct $\Phi_{n}(x)$ by the primary $\mathcal{O}$ and its scalar descendants $\square^{m} \mathcal{O}$. From the conformal transformation rules of the primary we can deduce that

$$
\begin{aligned}
& {\left[P_{\mu}, \square^{n} \mathcal{O}(x)\right]=i \partial_{\mu} \square^{n} \mathcal{O}(x),} \\
& {\left[M_{\mu \nu}, \square^{n} \mathcal{O}(x)\right]=\left[i\left(x_{\mu} \partial_{\nu}-x_{\nu} \partial_{\mu}\right)+\Sigma_{\nu \rho}^{(\mathcal{O})}\right] \square^{n} \mathcal{O}(x),} \\
& {\left[D, \square^{n} \mathcal{O}(x)\right]=i\left(\Delta+2 n+x^{\mu} \partial_{\mu}\right) \square^{n} \mathcal{O}(x),} \\
& {\left[K_{\mu}, \mathcal{O}(x)\right]=\left[i\left(x^{2} \partial_{\mu}-2 x_{\mu} x^{\nu} \partial_{\nu}-2 x_{\mu} \Delta\right)-2 x^{\nu} \Sigma_{\mu \nu}^{(\mathcal{O})}\right]} \\
& \quad \cdot \mathcal{O}(x), \\
& {\left[K_{\mu}, \square^{n} \mathcal{O}(x)\right]=2 n[d-2(\Delta+n)] i \partial_{\mu} \square^{n-1} \mathcal{O}(x)} \\
& \quad-4 n \Sigma_{\mu \nu}^{(\mathcal{O})} \partial^{\nu} \square^{n-1} \mathcal{O}(x) \\
& \quad+\left[i\left(x^{2} \partial_{\mu}-2 x_{\mu} x^{\rho} \partial_{\rho}-2 x_{\mu}(\Delta+2 n)\right)-2 x^{\rho} \Sigma_{\mu \rho}^{(\mathcal{O})}\right] \\
& \quad \cdot \square^{n} \mathcal{O}(x)
\end{aligned}
$$$$
(n \geq 1) .
$$

Comparing with (14), it implies the unique identification

$$
\begin{aligned}
\Phi_{2 n+1} & =0, \\
\Phi_{2 n} & =\frac{(-1)^{n} \alpha^{n} \Gamma(\Delta-d / 2+1)}{4^{n} n ! \Gamma(\Delta-d / 2+n+1)} \square^{n} \mathcal{O} .
\end{aligned}
$$

In conclusion, up to an overall constant, our arguments show that the renormalized primary at energy scale $\mu=1 / z$ is given by

$$
\begin{aligned}
\mathcal{O}(x, z) & =Z\left(z^{2} \square\right) \mathcal{O}(x) \\
& ={ }_{0} F_{1}\left(; \Delta-\frac{d}{2}+1 ;-\frac{\alpha z^{2}}{4} \square\right) \mathcal{O}(x),
\end{aligned}
$$

and $z^{\Delta} \mathcal{O}(x, z)$ corresponds to a bulk scalar field $\phi(x, z)$. In the $\alpha=0$ limit, it actually comes back to the usual language of CFT.

We notice that $Z\left(z^{2} \square\right)$ obtained in (17) is nothing but the Fourier transformation of the solutions to the bulk free field equation with $\Phi(z \rightarrow 0) \sim z^{\Delta}$ behavior at the boundary. This construction is different from the one suggested in [5-10]. The approach there encountered only the $k^{2}=\vec{k}^{2}-w^{2}<0$ part of the bulk $\Phi(z \rightarrow 0) \sim z^{\Delta}$ modes, and thus it cannot be generalized to the Euclidean AdS case. Instead, our construction encounters all the bulk $\Phi(z \rightarrow 0) \sim z^{\Delta}$ modes since it is the honest Fourier transformation. Obviously, (17) is applicable for both signatures.

2.2. Two-Point Correlators: The Divergent Regime and the Regulator. As a consistency check, let us use (17) to recover the well-known bulk-boundary propagator. We find

$$
\begin{aligned}
z^{\Delta} & \left\langle\mathcal{O}(z, x) \mathcal{O}\left(x^{\prime}\right)\right\rangle \\
= & \left\langle z_{0}^{\Delta} F_{1}\left(; \Delta-\frac{d}{2}+1 ;-\frac{\alpha z^{2}}{4} \square\right) \mathcal{O}(x) \mathcal{O}\left(x^{\prime}\right)\right\rangle \\
& =\frac{z^{\Delta}}{\left(x-x^{\prime}\right)^{2 \Delta}} \sum_{n=0}^{\infty} \frac{\Delta(\Delta+1) \cdots(\Delta+n-1)}{n !} \\
& \cdot\left(\frac{-\alpha z^{2}}{\left(x-x^{\prime}\right)^{2}}\right)^{n} .
\end{aligned}
$$

In the regime $\left|x-x^{\prime}\right|^{2}>\alpha z^{2}$, the series is convergent and gives rise to the expected form of the bulk-boundary propagator

$$
K\left(x, z ; x^{\prime}\right)=\left(\frac{z}{\alpha z^{2}+\left(x-x^{\prime}\right)^{2}}\right)^{\Delta} .
$$

However, in the regime $\left|x-x^{\prime}\right|^{2}<\alpha z^{2}$, the series (18) is divergent. In fact, this result is not surprising. The $Z\left(z^{2} \square\right)$ given in (17) is just the $\Phi(z \rightarrow 0) \sim z^{\Delta}$ modes of the bulk solution, while the Fourier transformation of (19) is a linear combination of the $\Phi(z \rightarrow 0) \sim z^{\Delta}$ modes and the $\Phi(z \rightarrow 0) \sim z^{d-\Delta}$ modes [14] which regulate the divergence of (18). The existence of the $\Phi(z \rightarrow 0) \sim z^{d-\Delta}$ constituent in (19) can be easily seen from the $z \rightarrow 0$ limit [15]

$$
\lim _{z \rightarrow 0}\left(\frac{z}{\alpha z^{2}+\left(x-x^{\prime}\right)^{2}}\right)^{\Delta} \sim z^{d-\Delta} \delta\left(x-x^{\prime}\right) .
$$

Although both the $\Phi(z \rightarrow 0) \sim z^{\Delta}$ and $\Phi(z \rightarrow 0) \sim z^{d-\Delta}$ modes diverge exponentially as $z \rightarrow \infty$, the combination is well-behaved in the interior since the two divergences cancel with each other. The explicit computation of the corresponding Fourier transformations is performed in Appendix.

In order to understand the above issue better, let us recall a simple fact in field theory. That is, the correlation function for composite operators always has zeroth-order UV divergence due to its composite natural. For example, 
consider the composite operator $\mathcal{O}=: \phi_{a} \phi^{a}$ :. The two-point correlator $\langle\mathcal{O O}\rangle$ receives zeroth-order UV divergence from the following loop diagram even in the free theory.

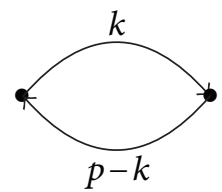

In the coordinate space, the corresponding divergence takes the following form:

$$
f(\Lambda, \mu, \square) \delta^{d}\left(x-x^{\prime}\right),
$$

where $\mu$ is the renormalization scale and $\Lambda$ is the cutoff scale. This divergence cannot be canceled by any local counterterm in the original action. Instead, we need to define the regularized two-point function directly and remove it by hand. Or equivalently speaking, we need to add a local counterterm in the free energy $W[J]$

$$
\begin{aligned}
e^{W[J]} & =\left\langle e^{J \mathscr{O}}\right\rangle, \\
W[J] & \longrightarrow W_{R}[J] \\
& =W[J]-\int \mathrm{d}^{d} x \int \mathrm{d}^{d} x^{\prime} c\left(x-x^{\prime}\right) J(x) J\left(x^{\prime}\right),
\end{aligned}
$$

where $c\left(x-x^{\prime}\right)=c(\Lambda, \mu, \square) \delta^{d}\left(x-x^{\prime}\right)$. In principle, after the cancelation of the divergent part, a possible remnant term in the form $R(\mu, \square) \delta^{d}\left(x-x^{\prime}\right)$ would still be there, and its explicit form depends on the prescription of the regularization.

We notice that the $\Phi(z \rightarrow 0) \sim z^{d-\Delta}$ modes of (19) in the coordinate space are given by

$$
z^{d-\Delta}{ }_{0} F_{1}\left(; \frac{d}{2}-\Delta+1 ;-\frac{\alpha z^{2}}{4} \square\right) \delta^{d}\left(x-x^{\prime}\right) .
$$

It is right in the form of $R(\mu, \square) \delta^{d}\left(x-x^{\prime}\right)$ appearing above. This fact suggests that one can understand it as the possible remnant term. The only special point is that there is an infinite order derivative operator acting on $\delta^{d}\left(x-x^{\prime}\right)$. Thus it is no longer a local function but a quasi-local term which is identically vanishing in the outer region $\left|x-x^{\prime}\right|^{2}>\alpha z^{2}$. Adding such a term does not affect the result (18) in the region $\left|x-x^{\prime}\right|^{2}>\alpha z^{2}$, and it is possible to cancel the divergence in the region $\left|x-x^{\prime}\right|^{2}<\alpha z^{2}$. If we take the continuity at $\left|x-x^{\prime}\right|^{2}=\alpha z^{2}$ as the prescription of the regularization of the two-point function, it will pick the correct ratio between the $\Phi(z \rightarrow 0) \sim z^{\Delta}$ modes and the $\Phi(z \rightarrow 0) \sim z^{d-\Delta}$ modes and then recovers (19) everywhere. This prescription is equivalent to the momentum space IR regularity condition used in the literatures [14]. Since it is natural to expect that effective operators defined at finite energy scale have some ambiguity in probing the distance shorter than its typical scale, the dependence on the prescription of regularization above is actually acceptable.
One can also check that the bulk-bulk propagator can be recovered by computing

$$
z^{\Delta} z^{\prime \Delta}\left\langle\mathcal{O}(z, x) \mathcal{O}\left(z^{\prime}, x^{\prime}\right)\right\rangle .
$$

Again, there is a divergent regime at short distance. If the continuity prescription is imposed, it implies that one should take the following regulator:

$$
\begin{aligned}
& z_{M}^{d-\Delta} z_{m 0}^{\Delta} F_{1}\left(; \frac{d}{2}-\Delta+1 ;-\frac{\alpha z_{M}^{2}}{4} \square\right) \\
& \cdot{ }_{0} F_{1}\left(; \Delta-\frac{d}{2}+1 ;-\frac{\alpha z_{m}^{2}}{4} \square\right) \delta^{d}\left(x-x^{\prime}\right),
\end{aligned}
$$

where $z_{M}=\max \left\{z, z^{\prime}\right\}$ and $z_{m}=\min \left\{z, z^{\prime}\right\}$.

\section{Radial Quantization}

3.1. Radial Quantization in CFT. In Section 2, we have constructed the bulk local operator and also explained its divergent regime with the regularization prescription there. However, the present formula is not convenient in discussing the bulk physics since the regulator should always be added by hand. It will be pretty nice if one can find a smart formula in which the regulator has been automatically built in. To achieve such a formula, let us discuss the radial quantization in usual CFT language firstly.

The radial quantization for CFT was detailedly reviewed in $[16,17]$. We will equivalently reexpress the results there by introducing the radial expansion for the operators. Again, we will just consider scalar primary here. The radial expansion of a scalar primary operator $\mathcal{O}(x)$ is given by

$$
\begin{aligned}
\mathcal{O}(x)= & \sum_{m=0}^{\infty} \frac{1}{m !} x^{\mu_{1}} \cdots x^{\mu_{m}} O_{\mu_{1} \cdots \mu_{m}} \\
& +\sum_{n=0}^{\infty} \frac{1}{n !} \check{x}^{2 \Delta} \check{x}^{\nu_{1}} \cdots \check{x}^{\nu_{n}} \check{O}_{\nu_{1} \cdots \nu_{n}},
\end{aligned}
$$

where $\check{x}$ is the inversion of $x$

$$
\check{x}^{\mu}=\mathscr{I} \circ x^{\mu}=\frac{x^{\mu}}{x^{2}} .
$$

In a unitary theory, its Hermitian conjugation is induced by the inversion

$$
\begin{aligned}
\mathcal{O}^{\dagger}(x)= & \check{O}(-x)=\frac{1}{x^{2 \Delta}} \mathcal{O}(-\check{x}) \\
= & \sum_{n=0}^{\infty} \frac{(-1)^{n}}{n !} x^{\nu_{1}} \cdots x^{\nu_{n}} \check{O}_{v_{1} \cdots \nu_{n}} \\
& +\sum_{m=0}^{\infty} \frac{(-1)^{m}}{m !} \check{x}^{2 \Delta} \check{x}^{\mu_{1}} \cdots \check{x}^{\mu_{m}} O_{\mu_{1} \cdots \mu_{m}} .
\end{aligned}
$$

In terms of the component operators, it is given by

$$
\begin{aligned}
& O_{\mu_{1} \cdots \mu_{n}}^{\dagger}=(-1)^{n} \check{O}_{\mu_{1} \cdots \mu_{n}}, \\
& \check{O}_{\mu_{1} \cdots \mu_{n}}^{\dagger}=(-1)^{n} O_{\mu_{1} \cdots \mu_{n}} .
\end{aligned}
$$


The vacuum $|1\rangle$ is defined by

$$
\begin{aligned}
\check{O}_{\nu_{1} \cdots \nu_{n}}|1\rangle & =0, \\
\langle 1| O_{\mu_{1} \cdots \mu_{m}} & =0 .
\end{aligned}
$$

It is equivalent to requiring that the state $\mathcal{O}(x)|1\rangle$ and all its descendants are regular at $x=0$, while the conjugated state $\langle 1| \check{O}(x)$ and all its descendants are also regular at $x=0$. One can check that the vacuum $|1\rangle$ defined in (30) is actually conformal invariant. The conformal transformation rules of the component operators can be deduced from the standard rules for primary $\mathcal{O}(x)$. We find

$$
\begin{aligned}
& {\left[P_{\mu}, O_{\mu_{1} \cdots \mu_{m}}\right]=i O_{\mu \mu_{1} \cdots \mu_{m}},} \\
& {\left[P_{\mu}, \check{O}_{v_{1} \cdots v_{n}}\right]=i\left[n(n-1) \eta_{\left(v_{1} v_{2}\right.} \check{O}_{\left.v_{3} \cdots v_{n}\right) \mu}\right.} \\
& \left.-2 n(\Delta+n-1) \eta_{\mu\left(v_{1}\right.} \check{O}_{\left.v_{2} \cdots v_{n}\right)}\right] \\
& {\left[M_{\mu \nu}, O_{\mu_{1} \cdots \mu_{m}}\right]=i m\left[\eta_{\mu\left(\mu_{1}\right.} O_{\left.\mu_{2} \cdots \mu_{m}\right) v}-\eta_{\nu\left(\mu_{1}\right.} O_{\left.\mu_{2} \cdots \mu_{m}\right) \mu}\right] \text {, }} \\
& {\left[M_{\mu \nu}, \check{O}_{v_{1} \cdots v_{n}}\right]=i n\left[\eta_{\mu\left(v_{1}\right.} \check{O}_{\left.v_{2} \cdots v_{n}\right) \nu}-\eta_{\nu\left(v_{1}\right.} \check{O}_{\left.v_{2} \cdots \nu_{m}\right) \mu}\right] \text {, }} \\
& {\left[D, O_{\mu_{1} \cdots \mu_{m}}\right]=i(\Delta+m) O_{\mu_{1} \cdots \mu_{m}},} \\
& {\left[D, \check{O}_{v_{1} \cdots v_{n}}\right]=-i(\Delta+m) \check{O}_{v_{1} \cdots \nu_{n}},} \\
& {\left[K_{\mu}, O_{\mu_{1} \cdots \mu_{m}}\right]=i\left[m(m-1) \eta_{\left(\mu_{1} \mu_{2}\right.} O_{\left.\mu_{3} \cdots \mu_{m}\right) \mu}\right.} \\
& \left.-2 m(\Delta+m-1) \eta_{\mu\left(\mu_{1}\right.} O_{\left.\mu_{2} \cdots \mu_{m}\right)}\right], \\
& {\left[K_{\mu}, \check{O}_{v_{1} \cdots v_{n}}\right]=i \check{O}_{\mu \nu_{1} \cdots v_{n}} \text {. }}
\end{aligned}
$$

Given the input data

$$
\left\langle\mathcal{O}^{\prime}(0) \mid \mathcal{O}(0)\right\rangle=\left\langle 1\left|\check{O}_{0}^{\prime} O_{0}\right| 1\right\rangle=C_{\mathscr{O}^{\prime} \mathscr{O}}
$$

one can decide the inner product between the states $\left|\partial_{\mu_{n}} \cdots \partial_{\mu_{1}} \mathcal{O}(0)\right\rangle=O_{\mu_{1} \cdots \mu_{n}}|1\rangle$ by using (31) and the conformal invariance of the vacuum. The result is

$$
\begin{aligned}
& \left\langle 1\left|\check{O}_{v_{n} \cdots \nu_{1}}^{\prime} O^{\mu_{1} \cdots \mu_{m}}\right| 1\right\rangle \\
& =\delta_{m n} C_{\mathcal{O}^{\prime} \mathcal{O}} \sum_{k=0}^{\lfloor n / 2\rfloor} \frac{(-1)^{n-k} 2^{n}(n !)^{2} \Gamma(\Delta+n-k)}{(n-2 k) ! k !(2 !)^{2 k} \Gamma(\Delta)} \delta_{\left(v_{1}\right.}^{\left(\mu_{1}\right.} \\
& \cdots \delta_{v_{n-2 k}}^{\mu_{n-2 k}} \eta_{v_{n-2 k+1} v_{n-2 k+2}} \cdots \eta_{\left.v_{n-1} \nu_{n}\right)} \eta^{\mu_{n-2 k+1} \mu_{n-2 k+2}} \\
& \cdots \eta^{\left.\mu_{n-1} \mu_{n}\right)} .
\end{aligned}
$$

Now we can reproduce the well-known two-point correlator by the "silly" computation

$$
\begin{aligned}
& \left\langle 1\left|\mathcal{O}(x) \mathcal{O}^{\prime}\left(x^{\prime}\right)\right| 1\right\rangle=\sum_{m=0}^{\infty} \sum_{n=0}^{\infty} \frac{1}{m ! n !} \\
& \cdot \frac{x^{\mu_{1}} \cdots x^{\mu_{m}} x^{\prime \nu_{1}} \cdots x^{\prime \nu_{n}}}{x^{2(\Delta+m)}}\left\langle 1\left|\check{O}_{\mu_{1} \cdots \mu_{m}} O_{v_{1} \cdots \nu_{n}}^{\prime}\right| 1\right\rangle \\
& =C_{\mathcal{O}^{\prime} \mathcal{O}} \sum_{\widehat{n}=0}^{\infty} \sum_{k=0}^{\infty} \frac{(-1)^{\widehat{n}+k} 2^{\widehat{n}} \Gamma(\Delta+\widehat{n}+k)}{\widehat{n} ! k ! \Gamma(\Delta)} \\
& \frac{\left(x \cdot x^{\prime}\right)^{\hat{n}} x^{\prime 2 k}}{x^{2(\Delta+\widehat{n}+k)}} \text {. }
\end{aligned}
$$

The convergence of the series requires that $|x|>\left|x^{\prime}\right|$. (Strictly speaking, the convergence argument is accurate only for the Euclidean case. For the Lorentzian case, proper analytical continuations are needed as what usually happened in the quantum field theory computations.) It means that the usual CFT correlator is reproduced by the radial ordered function $\left\langle 1\left|\widehat{\mathscr{R}} \mathcal{O}(x) \mathcal{O}^{\prime}\left(x^{\prime}\right)\right| 1\right\rangle$. In the radial quantization where the dilatation operator $D$ is treated as the Hamiltonian, the radial ordered function is the natural analogy of the time ordered function in the usual quantum field theory.

3.2. Radial Quantization at Finite Energy Scale. Now let us consider the CFT radial quantization in the presence of the finite energy scale $\mu$. A direct idea is acting upon the radial expansion (26) with the $Z\left(\mu^{-2} \square\right)$ in (17). We get

$$
\begin{aligned}
{ }_{0} F_{1} & \left(; \Delta-\frac{d}{2}+1 ;-\frac{\alpha z^{2}}{4} \square\right) \mathcal{O}(x) \\
& =\sum_{s=0}^{\infty} \sum_{k=0}^{\infty} \frac{\left(-\alpha z^{2}\right)^{k} \Gamma(\Delta-d / 2+1)}{2^{2 k} k ! s ! \Gamma(\Delta-d / 2+k+1)} \\
& \cdot x^{\nu_{1}} \cdots x^{\nu_{s}} O_{\mu_{1} \cdots \mu_{k}}{ }^{\mu_{1} \cdots \mu_{k}}{ }_{\nu_{1} \cdots \nu_{s}} \\
+ & \sum_{s=0}^{\infty} \sum_{l=0}^{\infty} \frac{\left(-\alpha z^{2}\right)^{l} \Gamma(\Delta-d / 2+1)}{2^{2 l} l ! s ! \Gamma(\Delta-d / 2+l+1)} \\
& \cdot{ }_{1} F_{0}\left(\Delta+2 l+s ; ;-\frac{\alpha z^{2}}{x^{2}}\right) \frac{x^{\nu_{1}} \cdots x^{\nu_{s}}}{x^{2(\Delta+2 l+s)}} \\
& \check{O}_{\mu_{1} \cdots \mu_{l}} \mu_{\nu_{1} \cdots \mu_{l}} \nu_{1} \cdots v_{s} \cdot
\end{aligned}
$$

We notice that when $|x|^{2}>\alpha z^{2}$

$$
\begin{aligned}
& \frac{1}{x^{2(\Delta+2 l+s)}}{ }_{1} F_{0}\left(\Delta+2 l+s ; ;-\frac{\alpha z^{2}}{x^{2}}\right) \\
& =\frac{1}{\left(x^{2}+\alpha z^{2}\right)^{\Delta+2 l+s}}
\end{aligned}
$$

and it is divergent in the regime $|x|^{2}<\alpha z^{2}$. The structure of the divergent regime is quite similar to what we have seen 
in Section 2. Thus it is natural to expect the following as the regularized radial expansion at finite energy scale

$$
\begin{aligned}
\mathcal{O}(x, z)= & \sum_{m=0}^{\infty} \frac{1}{m !} x^{\mu_{1}} \cdots x^{\mu_{m}} O_{\mu_{1} \cdots \mu_{m}}(z) \\
& +\sum_{n=0}^{\infty} \frac{1}{n !} \frac{x^{\nu_{1}} \cdots x^{\nu_{n}}}{\left(x^{2}+\alpha z^{2}\right)^{\Delta+n}} \check{O}_{\nu_{1} \cdots \nu_{n}}(\check{z}),
\end{aligned}
$$

where the component operator at finite energy scale is a linear combination of the original ones

$$
\begin{aligned}
& O_{\mu_{1} \cdots \mu_{m}}(z) \\
& \quad=\sum_{k=0}^{\infty} \frac{\left(-\alpha z^{2}\right)^{k} \Gamma(\Delta-d / 2+1)}{2^{2 k} k ! \Gamma(\Delta-d / 2+k+1)} O_{\nu_{1} \cdots v_{k}}^{\nu_{1} \cdots \nu_{k}}{ }_{\mu_{1} \cdots \mu_{m}}, \\
& \check{O}_{\mu_{1} \cdots \mu_{m}}(\check{z}) \\
& \quad=\sum_{k=0}^{\infty} \frac{\left(-\alpha \check{z}^{2}\right)^{k} \Gamma(\Delta-d / 2+1)}{2^{2 k} k ! \Gamma(\Delta-d / 2+k+1)} \check{O}_{\nu_{1} \cdots \nu_{k}}^{\nu_{1} \cdots \nu_{k}}{ }_{\mu_{1} \cdots \mu_{m}},
\end{aligned}
$$

and the inversion of $x$ and $z$ in the generalized scene is given by

$$
\begin{aligned}
& \check{x}^{\mu}=\mathscr{I} \circ x^{\mu}=\frac{x^{\mu}}{x^{2}+\alpha z^{2}}, \\
& \check{z}=\mathscr{I} \circ z=\frac{z}{x^{2}+\alpha z^{2}} .
\end{aligned}
$$

Correspondingly, the inversion of $\mathcal{O}(x, z)$ is

$$
\begin{aligned}
\check{O}(x, z)= & \frac{1}{\left(x^{2}+\alpha z^{2}\right)^{\Delta}} \mathcal{O}(\check{x} ; \check{z}) \\
= & \sum_{n=0}^{\infty} \frac{1}{n !} x^{\nu_{1}} \cdots x^{\nu_{n}} \check{O}_{\nu_{1} \cdots \nu_{n}}(z) \\
& +\sum_{m=0}^{\infty} \frac{1}{m !} \frac{x^{\mu_{1}} \cdots x^{\mu_{m}}}{\left(x^{2}+\alpha z^{2}\right)^{\Delta+n}} O_{\mu_{1} \cdots \mu_{m}}(\check{z}) .
\end{aligned}
$$

From (29), we can see that the Hermitian conjugation relation keeps intact at finite energy scale

$$
\mathcal{O}^{\dagger}(x, z)=\check{O}(-x, z)
$$

Simply by using (31), one can write down the conformal transformation rule for the component fields at finite energy scale directly as follows:

$$
\begin{aligned}
& {\left[P_{\mu}, O_{\mu_{1} \cdots \mu_{m}}(z)\right]=i O_{\mu \mu_{1} \cdots \mu_{m}}(z),} \\
& {\left[P_{\mu}, \check{O}_{\mu_{1} \cdots \mu_{m}}(z)\right]=i\left[\alpha z^{2} \check{O}_{\mu_{1} \cdots \mu_{m} \mu}(z)\right.} \\
& +m(m-1) \eta_{\left(\mu_{1} \mu_{2}\right.} \check{O}_{\left.\mu_{3} \cdots \mu_{m}\right) \mu}(z) \\
& \left.-2 m\left(\Delta+m-1+z \partial_{z}\right) \eta_{\mu\left(\mu_{1}\right.} \check{O}_{\left.\mu_{2} \cdots \mu_{m}\right)}(z)\right], \\
& {\left[M_{\mu \nu}, O_{\mu_{1} \cdots \mu_{m}}(z)\right]=i m\left[\eta_{\mu\left(\mu_{1}\right.} O_{\left.\mu_{2} \cdots \mu_{m}\right) \nu}(z)\right.} \\
& \left.-\eta_{\nu\left(\mu_{1}\right.} O_{\left.\mu_{2} \cdots \mu_{m}\right) \mu}(z)\right] \text {, } \\
& {\left[M_{\mu \nu}, \check{O}_{\mu_{1} \cdots \mu_{m}}(z)\right]=i m\left[\eta_{\mu\left(v_{1}\right.} \check{O}_{\left.v_{2} \cdots v_{m}\right) v}(z)\right.} \\
& \left.-\eta_{\nu\left(v_{1}\right.} \check{O}_{\left.v_{2} \cdots v_{m}\right) \mu}(z)\right] \text {, } \\
& {\left[D, O_{\mu_{1} \cdots \mu_{m}}(z)\right]=i\left(\Delta+m+z \partial_{z}\right) O_{\mu_{1} \cdots \mu_{m}}(z),} \\
& {\left[D, \check{O}_{\mu_{1} \cdots \mu_{m}}(z)\right]=-i\left(\Delta+m+z \partial_{z}\right) \check{O}_{\mu_{1} \cdots \mu_{m}}(z) \text {, }} \\
& {\left[K_{\mu}, O_{\mu_{1} \cdots \mu_{m}}(z)\right]=i\left[\alpha z^{2} O_{\mu_{1} \cdots \mu_{m} \mu}(z)\right.} \\
& +m(m-1) \eta_{\left(\mu_{1} \mu_{2}\right.} O_{\left.\mu_{3} \cdots \mu_{m}\right) \mu}(z) \\
& \left.-2 m\left(\Delta+m-1+z \partial_{z}\right) \eta_{\mu\left(\mu_{1}\right.} O_{\left.\mu_{2} \cdots \mu_{m}\right)}(z)\right], \\
& {\left[K_{\mu}, \check{O}_{\mu_{1} \cdots \mu_{m}}(z)\right]=i \check{O}_{\mu \mu_{1} \cdots \mu_{m}}(z) \text {. }}
\end{aligned}
$$

Similar to the arguments in Section 2.1, we can show that a bulk scalar field $\Phi(x, z)$ expanded as

$$
\begin{aligned}
\Phi(x, z)= & \sum_{m=0}^{\infty} \frac{z^{\Delta}}{m !} x^{\mu_{1}} \cdots x^{\mu_{m}} \Phi_{\mu_{1} \cdots \mu_{m}}(z) \\
& +\sum_{n=0}^{\infty} \frac{1}{n !} \frac{z^{\Delta} x^{\nu_{1}} \cdots x^{\nu_{n}}}{\left(x^{2}+\alpha z^{2}\right)^{\Delta+n}} \check{\Phi}_{v_{1} \cdots v_{n}}(\check{z})
\end{aligned}
$$

indeed requires the transformation rules (42) for its components. Thus, the regularized version (37) of primary at finite energy scale satisfies our basic renormalization condition for primary operators.

Parallel with (34), the standard bulk-boundary as well as bulk-bulk propagator can be reproduced by computing the radial ordered function $\left\langle 1\left|\widehat{\mathscr{R}} O(x, z) \mathcal{O}^{\prime}\left(x^{\prime}, z^{\prime}\right)\right| 1\right\rangle$. In our present case where energy scales are introduced, the radial order is defined by $x^{2}+\alpha z^{2}$. Providing this radial order, there is no ambiguity everywhere in reproducing the bulkboundary and bulk-bulk propagator. Therefore, (37) is indeed the smart formula which we are looking for.

Since our construction directly comes back to the standard CFT language in the $\alpha \rightarrow 0$ limit, it is possible that (37) will not suffer from the problem about bulk locality appearing in the smearing operator construction [18]. In order to address it properly, one should generalize the standard results 
about OPE to the cases with finite energy scales. We hope to report on this issue in a future work.

Formula (37) also suggests that one could define the finite energy scale effective Hilbert space $\mathscr{H}_{z}$ by acting on the vacuum $|1\rangle$ with $O_{\mu_{1} \cdots \mu_{m}}(z)$. An interesting observation is that $\mathscr{H}_{z}$ actually contains less information than the UV Hilbert space $\mathscr{H}=\mathscr{H}_{z=0}$. For example, considering the scalar sector, one can construct the following state:

$|\lambda\rangle$

$$
\begin{aligned}
& =\sum_{n=0}^{\infty} \frac{\lambda^{n} \Gamma(\Delta) \Gamma(\Delta-d / 2+1) \Gamma(d / 2)}{2^{4 n} n ! \Gamma(\Delta+n) \Gamma(\Delta-d / 2+n+1) \Gamma(n+d / 2)} \\
& \cdot O_{n}|1\rangle,
\end{aligned}
$$

where we denote $O_{n}(z)=O_{v_{1} \cdots v_{n}}{ }_{1} \cdots v_{n}(z)$. It is a well-defined state in $\mathscr{H}$ since the norm is finite

$$
=C_{\mathscr{O}} \sum_{n=0}^{\infty} \frac{\lambda^{n} \Gamma(\Delta) \Gamma(\Delta-d / 2+1) \Gamma(d / 2)}{2^{4 n} n ! \Gamma(\Delta+n) \Gamma(\Delta-d / 2+n+1) \Gamma(n+d / 2)} .
$$

The inner product between the state $|\lambda\rangle$ and the states in $\mathscr{H}_{z}$ is given by

$$
\left\langle\lambda\left|O_{m}(z)\right| 1\right\rangle=C_{\mathcal{O}} \lambda^{m} \sum_{k=0}^{\infty} \frac{\left(-\alpha \lambda z^{2}\right)^{k} \Gamma(\Delta-d / 2+1)}{2^{2 k} k ! \Gamma(\Delta-d / 2+k+1)} .
$$

Since the ${ }_{0} F_{1}$ function has infinite number of zeros, the inner product will be zero for infinite many $\lambda$ 's providing $z \neq 0$. Therefore, there are an infinite number of states in $\mathscr{H}$ perpendicular to the finite energy scale effective Hilbert space $\mathscr{H}_{z \neq 0}$. Although this observation is something one could expect for effective descriptions at finite energy scale, it may have some possible advantages in discussing the $c$-theorem and the entanglement entropy.

\section{Discussions}

In the previous sections, we suggest a CFT construction of the bulk local operators in pure AdS space. The construction is based on considering CFT at finite energy scale. The basic result is that bulk operator is given by acting upon the original CFT primary with an infinite order differential operator $Z_{\text {AdS }}(\mu, \partial)$. Although we do not assume any knowledge about the bulk in advance, our arguments automatically show that $Z_{\text {AdS }}(\mu, \partial)$ should be the Fourier transformation of the $\Phi(z \rightarrow$ $0) \sim z^{\Delta}$ solution to the bulk free field equation. We also discuss the relation between the regulator of the two-point function and the $\Phi(z \rightarrow 0) \sim z^{d-\Delta}$ modes. In Section 3, based on the radial quantization in CFT, we find an improved formula of our construction in which the regulator is automatically built in.

The next challenge is how to generalize our construction to geometries beyond pure AdS. A naive guess is that the bulk local operator is also effectively given by acting upon the original CFT primary with the infinite order differential operator
$Z_{\text {Geom }}(\mu, \partial)$, which is the Fourier transformation of the bulk $\Phi(z \rightarrow 0) \sim z^{\Delta}$ modes in the corresponding geometry. On the other hand, our CFT arguments in Section 2.1 seem state independent. Thus, it suggests that the bulk local operator should always be given by $Z_{\text {AdS }}(\mu, \partial)$ for all asymptotic AdS geometries which are basically very heavy excited states in the CFT. We conjecture that these two possibilities are actually complementary to each other. The explicit proposal [19] for the underlying mechanism can be summarized as the following.

Bulk geometries are actually dual to the coherent states $\mid$ Geom $\rangle=F\left(T_{\mu \nu}\right)|1\rangle$ which is created by acting on the vacuum $|1\rangle$ with certain function $F\left(T_{\mu \nu}\right)$ of stress tensor and its descendants.

The bulk correlators of the dual field $\phi(x, z)$ can be reproduced by computing

$$
\begin{aligned}
& \left\langle\text { Geom }\left|\cdots Z_{\text {AdS }}(\mu, \partial) \mathcal{O}(x) \cdots\right| \text { Geom }\right\rangle \\
& \quad=\left\langle 1\left|F^{\dagger}\left(T_{\mu \nu}\right) \cdots Z_{\text {AdS }}(\mu, \partial) \mathcal{O}(x) \cdots F\left(T_{\mu \nu}\right)\right| 1\right\rangle .
\end{aligned}
$$

On the other hand, by using the local conformal Ward identity [20-22], one may convert (at least in the two-dimensional CFT) the effects of $F\left(T_{\mu \nu}\right)$ to a differential operator $\widehat{\mathscr{F}}[F]$ acting on the operator $Z_{\mathrm{AdS}}(\mu, \partial) \mathcal{O}(x)$ as follows:

$$
\begin{gathered}
\left\langle 1\left|F^{\dagger}\left(T_{\mu \nu}\right) \cdots Z_{\text {AdS }}(\mu, \partial) \mathcal{O}(x) \cdots F\left(T_{\mu \nu}\right)\right| 1\right\rangle \\
=\left\langle 1\left|\cdots \widehat{\mathscr{F}}[F] Z_{\text {AdS }}(\mu, \partial) \mathcal{O}(x) \cdots\right| 1\right\rangle .
\end{gathered}
$$

The new differential operator $\widehat{\mathscr{F}}[F] Z_{\text {AdS }}(\mu, \partial)$ is expected to be exactly $Z_{\mathrm{Geom}}(\mu, \partial)$ of the corresponding geometry. For the black hole geometry, the horizon is the position where the series in $Z_{\text {Geom }}(\mu, \partial)$ becomes ill-defined. However, everything could be still well defined after coming back to the $\mid$ Geom $\rangle$ description and one can explore the black hole interior in this formalism.

Finally, it is also possible that multiple states with different $F\left(T_{\mu \nu}\right)$ give rise to the same differential operator $Z_{\text {Geom }}(\mu, \partial)$. Thus it could be a dual CFT way to explain the entropy of AdS black hole. If indeed so, it means that all the black hole microstates should correspond exactly to the same geometry, and thus one does not need to take any average over different microgeometries. This picture seems different from what people usually expected for quantum gravity and may offer new possibilities to the discussions of the black hole firewall problem $[23,24]$.

As the early version of this work was drawing a conclusion, [25] appeared with results which partially overlap with Section 2.1 in this manuscript. We also realized that the twodimensional version of their results has already appeared in [26]. 


\section{Appendix}

\section{A. The Momentum Space Formula}

In the momentum space, the general solutions to the bulk free scalar equation are given by the linear combination of the $\Phi(z \rightarrow 0) \sim z^{\Delta}$ modes

$$
\begin{aligned}
& z^{\Delta} k_{0}^{2 \Delta-d} F_{1}\left(; \Delta-\frac{d}{2}+1 ; \frac{z^{2} k^{2}}{4}\right) \\
& =\Gamma\left(\Delta-\frac{d}{2}+1\right) z^{d / 2}(2 k)^{\Delta-d / 2} I_{\Delta-d / 2}
\end{aligned}
$$

as well as the $\Phi(z \rightarrow 0) \sim z^{d-\Delta}$ modes

$$
\begin{aligned}
z^{d-\Delta}{ }_{0} F_{1}\left(; \frac{d}{2}-\Delta+1 ; \frac{z^{2} k^{2}}{4}\right) \\
\quad=\Gamma\left(\frac{d}{2}-\Delta+1\right) z^{d / 2}\left(\frac{k}{2}\right)^{\Delta-d / 2} I_{d / 2-\Delta} .
\end{aligned}
$$

In the following, let us derive the Fourier transformation of them, respectively.

A.1. $\Phi(z \rightarrow 0) \sim z^{\Delta}$ Modes. The Fourier transformation of the $\Phi(z \rightarrow 0) \sim z^{\Delta}$ mode is

$$
\begin{aligned}
& \lim _{\Lambda \rightarrow \infty} \frac{z^{d / 2}}{(2 \pi)^{d}} \int \mathrm{d}^{d} k \mathrm{e}^{-(z k)^{2} / \Lambda^{2}} I_{\Delta-d / 2}(z k) k^{\Delta-d / 2} \mathrm{e}^{i k\left(x-x^{\prime}\right)} \\
& =\lim _{\Lambda \rightarrow \infty} \frac{z^{d / 2}(2 \pi)^{d}}{\Gamma((d-1) / 2)} \frac{2 \pi^{(d-1) / 2}}{\Gamma \pi((d-1) / 2)} \\
& \cdot \int_{0}^{\infty} \mathrm{d} k \mathrm{e}^{-(z k)^{2} / \Lambda^{2}} I_{\Delta-d / 2}(z k) \\
& \cdot k^{\Delta+d / 2-1}{ }_{0} F_{1}\left(; \frac{d}{2} ;-\frac{k^{2} r^{2}}{4}\right)=\lim _{\Lambda \rightarrow \infty} \frac{z^{d / 2}}{(2 \pi)^{d}} \\
& \cdot \frac{2 \pi^{(d-1) / 2}}{\Gamma((d-1) / 2)} \frac{\sqrt{\pi} \Gamma((d-1) / 2)}{\Gamma(d / 2)} \\
& \cdot \sum_{m=0}^{\infty} \frac{1}{m ! \Gamma(\Delta+m-d / 2+1)} \int_{0}^{\infty} \mathrm{d} k \\
& \cdot \mathrm{e}^{-(z k)^{2} / \Lambda^{2}}\left(\frac{z k}{2}\right)^{2 m+\Delta-d / 2} \\
& \cdot k^{\Delta+d / 2-1}{ }_{0} F_{1}\left(; \frac{d}{2} ;-\frac{k^{2} r^{2}}{4}\right)=\lim _{\Lambda \rightarrow \infty} \frac{1}{(2 \pi)^{d}} \\
& \cdot \frac{2 \pi^{(d-1) / 2}}{\Gamma((d-1) / 2)} \frac{\sqrt{\pi} \Gamma((d-1) / 2)}{2 \Gamma(d / 2)} \\
& \cdot \sum_{m=0}^{\infty} \frac{2^{-2 m-\Delta+d / 2} z^{-\Delta}}{m ! \Gamma(\Delta+m-d / 2+1)} \Gamma(\Delta+m) \\
& \cdot \Lambda^{2(\Delta+m)}{ }_{1} F_{1}\left(\Delta+m ; \frac{d}{2} ;-\frac{\Lambda^{2} r^{2}}{4 z^{2}}\right) \\
&
\end{aligned}
$$

where we have used the fact that the convergence radius of ${ }_{0} F_{1}$ and ${ }_{1} F_{1}$ is $\infty$ and

$$
\begin{aligned}
& \int_{0}^{\pi} \mathrm{d} \theta(\sin \theta)^{d-2} \mathrm{e}^{i k r \cos \theta} \\
& \quad=\frac{\sqrt{\pi} \Gamma((d-1) / 2)}{\Gamma(d / 2)}{ }_{0} F_{1}\left(; \frac{d}{2} ;-\frac{k^{2} r^{2}}{4}\right), \\
& \int_{0}^{\infty} \mathrm{d} k \mathrm{e}^{-k^{2} / \Lambda^{2}} k^{2 \Delta-1+2 n}=\frac{1}{2} \Lambda^{2(\Delta+n)} \Gamma(\Delta+n)
\end{aligned}
$$

$$
(\Delta+n>0) .
$$

By using the asymptotic expansion of confluent hypergeometric function

$$
\begin{aligned}
& { }_{1} F_{1}(\alpha ; \gamma ; z) \\
& =\frac{\Gamma(\gamma)}{\Gamma(\gamma-\alpha)} \mathrm{e}^{i \pi \alpha} z^{-\alpha}{ }_{2} F_{0}\left(\alpha, \alpha-\gamma+1 ; ;-z^{-1}\right) \\
& \quad+\frac{\Gamma(\gamma)}{\Gamma(\alpha)} \mathrm{e}^{z} z^{\alpha-\gamma}{ }_{2} F_{0}\left(\gamma-\alpha, 1-\alpha ; z^{-1}\right) \\
& \text { for }-\frac{\pi}{2}<\arg z<\frac{3 \pi}{2}
\end{aligned}
$$

we find in the regime $\left(x-x^{\prime}\right)^{2}>z^{2}$

$$
\begin{aligned}
& \lim _{\Lambda \rightarrow \infty} \frac{1}{(2 \pi)^{d}} \frac{2 \pi^{(d-1) / 2}}{\Gamma((d-1) / 2)} \frac{\sqrt{\pi} \Gamma((d-1) / 2)}{2 \Gamma(d / 2)} \\
& \quad \cdot \sum_{m=0}^{\infty} \frac{2^{-2 m-\Delta+d / 2} z^{-\Delta}}{m ! \Gamma(\Delta+m-d / 2+1)} \Gamma(\Delta+m) \\
& \cdot \Lambda^{2(\Delta+m)}{ }_{1} F_{1}\left(\Delta+m ; \frac{d}{2} ;-\frac{\Lambda^{2} r^{2}}{4 z^{2}}\right) \\
& \quad=\frac{2^{\Delta-d / 2} z^{-\Delta} \sin (((d-2 \Delta) \pi) / 2) \Gamma(\Delta)}{\pi^{d / 2+1}} \\
& \quad \cdot \sum_{m=0}^{\infty} \frac{(-1)^{m} \Gamma(\Delta+m)}{m ! \Gamma(\Delta)} \frac{z^{2(\Delta+m)}}{\left(x-x^{\prime}\right)^{2(\Delta+m)}} \\
& \quad=\frac{2^{\Delta-d / 2} \sin (((d-2 \Delta) \pi) / 2) \Gamma(\Delta)}{\pi^{d / 2+1}}\left(\frac{z}{z^{2}+\left(x-x^{\prime}\right)^{2}}\right)^{\Delta} .
\end{aligned}
$$

Thus the $\Phi(z \rightarrow 0) \sim z^{\Delta}$ mode reproduces $K\left(z, x ; x^{\prime}\right)$ for the region $\left(x-x^{\prime}\right)^{2}>z^{2}$ as expected.

For $\left(x-x^{\prime}\right)^{2}<z^{2}$, we exchange the order of summations and find

$$
\begin{aligned}
& \lim _{\Lambda \rightarrow \infty} \frac{1}{(2 \pi)^{d}} \frac{2 \pi^{(d-1) / 2}}{\Gamma((d-1) / 2)} \frac{\sqrt{\pi} \Gamma((d-1) / 2)}{2 \Gamma(d / 2)} \\
& \cdot \sum_{m=0}^{\infty} \frac{2^{-2 m-\Delta+d / 2} z^{-\Delta}}{m ! \Gamma(\Delta+m-d / 2+1)} \Gamma(\Delta+m) \\
& \cdot \Lambda^{2(\Delta+m)}{ }_{1} F_{1}\left(\Delta+m ; \frac{d}{2} ;\right. \\
& \left.-\frac{\Lambda^{2} r^{2}}{4 z^{2}}\right)=\lim _{\Lambda \rightarrow \infty} \frac{1}{(2 \pi)^{d}} \frac{2 \pi^{(d-1) / 2}}{\Gamma((d-1) / 2)}
\end{aligned}
$$




$$
\begin{aligned}
& \cdot \frac{\sqrt{\pi} \Gamma((d-1) / 2)}{2 \Gamma(d / 2)} \sum_{m=0}^{\infty} \sum_{n=0}^{\infty} \frac{2^{-2 m-\Delta+d / 2} z^{-\Delta}}{m ! \Gamma(\Delta+m-d / 2+1)} \\
& \cdot \frac{\Gamma(\Delta+m+n) \Gamma(d / 2)}{n ! \Gamma(d / 2+n)} \Lambda^{2(\Delta+m)}\left(-\frac{\Lambda^{2} r^{2}}{4 z^{2}}\right)^{n} \\
& =\lim _{\Lambda \rightarrow \infty} \frac{1}{(2 \pi)^{d}} \frac{2 \pi^{(d-1) / 2}}{\Gamma((d-1) / 2)} \frac{\sqrt{\pi} \Gamma((d-1) / 2)}{2 \Gamma(d / 2)} \\
& \cdot \sum_{n=0}^{\infty} \frac{2^{-\Delta+d / 2} \Lambda^{2 \Delta} z^{-\Delta}}{\Gamma(\Delta-d / 2+1)} \frac{\Gamma(\Delta+n) \Gamma(d / 2)}{n ! \Gamma(d / 2+n)}{ }_{1} F_{1}(\Delta \\
& \left.+n ; \Delta-\frac{d}{2}+1 ; \frac{\Lambda^{2}}{4}\right)\left(-\frac{\Lambda^{2} r^{2}}{4 z^{2}}\right)^{n}=\frac{1}{(2 \pi)^{d}} \\
& \cdot \frac{2 \pi^{(d-1) / 2}}{\Gamma((d-1) / 2)} \frac{\sqrt{\pi} \Gamma((d-1) / 2)}{2 \Gamma(d / 2)} \\
& \frac{2^{\Delta+d / 2} z^{-\Delta} \Gamma(\Delta) \sin (d \pi / 2) \Gamma(d / 2)}{\pi} \sum_{n=0}^{\infty} \frac{\Gamma(\Delta+n)}{n ! \Gamma(\Delta)} \\
& \cdot\left(-\frac{r^{2}}{z^{2}}\right)^{n}\left[\mathrm{e}^{i \pi \Delta}+\lim _{\Lambda \rightarrow \infty}(-1)^{n} \frac{\Gamma(1-d / 2-n)}{\Gamma(\Delta+n)}\right. \\
& \cdot \mathrm{e}^{\Lambda^{2} / 4}\left(\frac{\Lambda^{2}}{4}\right)^{\Delta+d / 2+2 n-1} \\
& \left.\cdot{ }_{2} F_{0}\left(1-\frac{d}{2}-n ; 1-\Delta-n ; \frac{4}{\Lambda^{2}}\right)\right] \\
& =\frac{2^{\Delta-d / 2} \Gamma(\Delta)}{\pi^{d / 2+1}} \mathrm{e}^{i \pi \Delta} \sin \left(\frac{d \pi}{2}\right)\left(\frac{z}{z^{2}+\left(x-x^{\prime}\right)^{2}}\right)^{\Delta} \\
& +\frac{2^{\Delta-d / 2}}{\pi^{d / 2}} \lim _{\Lambda \rightarrow \infty} \sum_{n=0}^{\infty} \frac{\left(-r^{2} / z^{2}\right)^{n}}{n ! \Gamma(d / 2+n)} \\
& \cdot \mathrm{e}^{\Lambda^{2} / 4}\left(\frac{\Lambda^{2}}{4}\right)^{\Delta+d / 2+2 n-1}{ }_{2} F_{0}\left(1-\frac{d}{2}-n ; 1-\Delta\right. \\
& \left.-n ; \frac{4}{\Lambda^{2}}\right) .
\end{aligned}
$$

The result is divergent and the divergent parts are expressed as terms with positive powers of $\Lambda$.

A.2. $\Phi(z \rightarrow 0) \sim z^{d-\Delta}$ Modes. For the $\Phi(z \rightarrow 0) \sim z^{\Delta}$ modes, in the regime $\left(x-x^{\prime}\right)^{2}>z^{2}$, we have

$$
\begin{gathered}
\lim _{\Lambda \rightarrow \infty} \frac{z^{d / 2}}{(2 \pi)^{d}} \int \mathrm{d}^{d} k \mathrm{e}^{-(z k)^{2} / \Lambda^{2}} I_{d / 2-\Delta}(z k) k^{\Delta-d / 2} \mathrm{e}^{i k\left(x-x^{\prime}\right)} \\
\quad=\lim _{\Lambda \rightarrow \infty} \frac{z^{d / 2}}{(2 \pi)^{d}} \frac{2 \pi^{(d-1) / 2}}{\Gamma((d-1) / 2)} \frac{\sqrt{\pi} \Gamma((d-1) / 2)}{\Gamma(d / 2)}
\end{gathered}
$$

$$
\begin{aligned}
& \cdot \int_{0}^{\infty} \mathrm{d} k \mathrm{e}^{-(z k)^{2} / \Lambda^{2}} I_{d / 2-\Delta}(z k) \\
& \cdot k^{\Delta+d / 2-1}{ }_{0} F_{1}\left(; \frac{d}{2} ;-\frac{k^{2} r^{2}}{4}\right)=\lim _{\Lambda \rightarrow \infty} \frac{z^{d / 2}}{(2 \pi)^{d}} \\
& \cdot \frac{2 \pi^{(d-1) / 2}}{\Gamma((d-1) / 2)} \frac{\sqrt{\pi} \Gamma((d-1) / 2)}{\Gamma(d / 2)} \\
& \cdot \sum_{m=0}^{\infty} \frac{1}{m ! \Gamma(-\Delta+m+d / 2+1)} \int_{0}^{\infty} \mathrm{d} k \mathrm{e}^{-(z k)^{2} / \Lambda^{2}} \\
& \cdot\left(\frac{z k}{2}\right)^{2 m-\Delta+d / 2} k^{\Delta+d / 2-1}{ }_{0} F_{1}\left(; \frac{d}{2} ;-\frac{k^{2} r^{2}}{4}\right) \\
& =\lim _{\Lambda \rightarrow \infty} \frac{1}{(2 \pi)^{d}} \frac{2 \pi^{(d-1) / 2}}{\Gamma((d-1) / 2)} \frac{\sqrt{\pi} \Gamma((d-1) / 2)}{2 \Gamma(d / 2)} \\
& \cdot \sum_{m=0}^{\infty} \frac{2^{-2 m+\Delta-d / 2} z^{-\Delta}}{m ! \Gamma(-\Delta+m+d / 2+1)} \Gamma\left(\frac{d}{2}+m\right) \\
& \cdot \Lambda^{d+2 m}{ }_{1} F_{1}\left(\frac{d}{2}+m ; \frac{d}{2} ;-\frac{\Lambda^{2} r^{2}}{4 z^{2}}\right) \\
& =\sum_{m=0}^{\infty} \frac{2^{-2 m+\Delta-d / 2} z^{-\Delta}}{m ! \Gamma(-\Delta+m+d / 2+1)} \frac{2^{m} \Gamma(d / 2+m)}{\pi^{d / 2} \Gamma(-m)} \\
& \frac{z^{d+2 m}}{\left(x-x^{\prime}\right)^{d+2 m}}=0
\end{aligned}
$$

where we have also used the asymptotic expansion (A.6) of confluent hypergeometric function ${ }_{1} F_{1}$. The above result shows that the $\Phi(z \rightarrow 0) \sim z^{d-\Delta}$ mode is a quasi-local function which is identically vanishing when $\left(x-x^{\prime}\right)^{2}>z^{2}$.

In the $\left(x-x^{\prime}\right)^{2}<z^{2}$ regime, we have

$$
\begin{aligned}
& \frac{z^{d / 2}}{(2 \pi)^{d}} \int \mathrm{d}^{d} k \mathrm{e}^{-(z k)^{2} / \Lambda^{2}} I_{d / 2-\Delta}(z k) k^{\Delta-d / 2} \mathrm{e}^{i k\left(x-x^{\prime}\right)} \\
& =\frac{2^{\Delta-d / 2} z^{d-\Delta}}{\Gamma(d / 2-\Delta+1)}{ }_{0} F_{1}\left(; \frac{d}{2}-\Delta+1 ;-\frac{z^{2}}{4} \square\right) \\
& \cdot \delta^{d}\left(x-x^{\prime}\right)=\lim _{\Lambda \rightarrow \infty} \frac{2^{\Delta-d / 2} z^{d-\Delta}}{\Gamma(d / 2-\Delta+1)}{ }_{0} F_{1}\left(; \frac{d}{2}\right. \\
& \left.-\Delta+1 ;-\frac{z^{2}}{4}\right) \frac{\Lambda^{d}}{2^{d} \pi^{d / 2} z^{d}} \mathrm{e}^{-\left(x-x^{\prime}\right)^{2} \Lambda^{2} / 4 z^{2}} \\
& =\lim _{\Lambda \rightarrow \infty} \frac{2^{\Delta-d / 2} z^{d-\Delta}}{\Gamma(d / 2-\Delta+1)} \frac{\Lambda^{d}}{2^{d} \pi^{d / 2} z^{d}{ }_{0} F_{1}}\left(; \frac{d}{2}-\Delta\right. \\
& \left.+1 ;-\frac{z^{2}}{4} \square\right) \sum_{m=0}^{\infty} \frac{1}{m !}\left(\frac{-\left(x-x^{\prime}\right)^{2} \Lambda^{2}}{4 z^{2}}\right)^{m} \\
& \quad=\lim _{\Lambda \rightarrow \infty} \frac{2^{\Delta-d / 2} z^{d-\Delta}}{\Gamma(d / 2-\Delta+1)} \frac{\Lambda^{d}}{2^{d} \pi^{d / 2} z^{d}}
\end{aligned}
$$




$$
\begin{aligned}
& \cdot \sum_{m=0}^{\infty} \sum_{n=0}^{m} \frac{\Gamma(d / 2-\Delta+1)}{n ! \Gamma(d / 2-\Delta+1+n)} \\
& \frac{\Gamma(d / 2+m)}{\Gamma(m-n+1) \Gamma(d / 2+m-n)}\left(\frac{\Lambda^{2}}{4}\right)^{m} \\
& \left(-\frac{\left(x-x^{\prime}\right)^{2}}{z^{2}}\right)^{(m-n)}=\lim _{\Lambda \rightarrow \infty} \frac{2^{\Delta-d / 2} z^{d-\Delta}}{\Gamma(d / 2-\Delta+1)} \\
& \frac{\Lambda^{d}}{2^{d} \pi^{d / 2} z^{d}} \sum_{k=0}^{\infty} \frac{1}{k !}{ }_{1} F_{1}\left(\frac{d}{2}+k ; \frac{d}{2}-\Delta+1 ; \frac{\Lambda^{2}}{4}\right) \\
& \cdot\left(-\frac{\Lambda^{2}\left(x-x^{\prime}\right)^{2}}{4 z^{2}}\right)^{k}=\frac{2^{\Delta-d / 2} z^{-\Delta}}{\pi^{d / 2}} \sum_{k=0}^{\infty}\left[\mathrm{e}^{i(d \pi / 2)}\right. \\
& \frac{\sin (\pi \Delta)}{\pi} \Gamma(\Delta) \frac{\Gamma(\Delta+k)}{k ! \Gamma(\Delta)}\left(-\frac{\left(x-x^{\prime}\right)^{2}}{z^{2}}\right)^{k} \\
& +\lim _{\Lambda \rightarrow \infty} \frac{\left(-\left(x-x^{\prime}\right)^{2} / z^{2}\right)^{k}}{k ! \Gamma(d / 2+k)} \mathrm{e}^{\Lambda^{2} / 4}\left(\frac{\Lambda^{2}}{4}\right)^{\Delta+d / 2+2 k-1} \\
& \left.\cdot{ }_{2} F_{0}\left(1-\Delta-k, 1-\frac{d}{2}-k ; \frac{4}{\Lambda^{2}}\right)\right] \\
& =\frac{2^{\Delta-d / 2} \Gamma(\Delta)}{\pi^{d / 2+1}} \mathrm{e}^{i(d \pi / 2)} \sin (\pi \Delta)\left(\frac{z}{z^{2}+\left(x-x^{\prime}\right)^{2}}\right)^{\Delta} \\
& +\lim _{\Lambda \rightarrow \infty} \frac{2^{\Delta-d / 2} z^{-\Delta}}{\pi^{d / 2}} \sum_{k=0}^{\infty} \frac{\left(-\left(x-x^{\prime}\right)^{2} / z^{2}\right)^{k}}{k ! \Gamma(d / 2+k)} \\
& \cdot \mathrm{e}^{\Lambda^{2} / 4}\left(\frac{\Lambda^{2}}{4}\right)^{\Delta+d / 2+2 k-1}{ }_{2} F_{0}\left(1-\Delta-k, 1-\frac{d}{2}\right. \\
& \left.-k ; \frac{4}{\Lambda^{2}}\right) \text {. }
\end{aligned}
$$

As in the $\Phi(z \rightarrow 0) \sim z^{\Delta}$ case, the result is divergent and the divergent parts are expressed as terms with positive powers of $\Lambda$.

Comparing (A.7) and (A.9), we notice that the divergence in the region $\left(x-x^{\prime}\right)^{2}<z^{2}$ can only be canceled in the combination

$$
I_{\Delta-d / 2}-I_{d / 2-\Delta} \sim K_{\Delta-d / 2},
$$

which coincides with the answer obtained in [14] by requiring the $z \rightarrow \infty$ regularity of the momentum space solution. We also notice that the remnant finite term gives rise to exactly the analytical continuation of the result in $\left(x-x^{\prime}\right)^{2}>z^{2}$ region. Thus one can take the continuity at $\left|x-x^{\prime}\right|^{2}=z^{2}$ as the prescription of the regularization in the coordinate space.
The above derivations are performed under the Euclidean signature. For the Minkowskian signature, the 2-point propagator is not unique due to the existence of the light cone singularity. Depending on which kind of 2-point propagator was considered, the corresponding bulk momentum space formulae are different. These different formulae are related to the different choices of quantum states of the boundary QFT [27, 28]. For example, as pointed in [29-31], the retarded propagator is related to take the ingoing boundary condition at the horizon $z \rightarrow \infty$, while the advanced propagator is related to take the outcoming boundary condition at the horizon $z \rightarrow \infty$. In these two cases, the relevant bulk momentum space formulae are still linear combinations of the $\Phi(z \rightarrow$ $0) \sim z^{\Delta}$ mode and the $\Phi(z \rightarrow 0) \sim z^{d-\Delta}$ mode. It is also possible to have the bulk momentum space formulae with purely normalizable modes where $\Phi(z \rightarrow 0) \sim z^{\Delta}$ and $k^{2}<0$. This case is in fact the one being discussed in [5-7]. In our present consideration, the formula is related to the radial quantization which is initially well established under the Euclidean signature. Therefore, the relevant bulk momentum space formula is taken to be the simple analytical continuation of the Euclidean one [14] which is uniquely fixed by requiring the regularity at $z \rightarrow \infty$ since $k^{2}>0$ for the Euclidean signature.

\section{Conflicts of Interest}

The authors declare that there are no conflicts of interest regarding the publication of this paper.

\section{Acknowledgments}

The authors are grateful to Piljin Yi for enlightening discussions as well as collaboration at an early stage of this work and also thanks are due to Jiang Long, Hong Lü, Jun-Bao Wu, Xiao Xiao, and Hossein Yavartanoo for useful conversations. This work is supported by National Natural Science Foundation of China with Grant no. 11305125 and no. 11447607 and the Double First-Class University Construction Project of Northwest University.

\section{References}

[1] J. Maldacena, "The Large N limit of superconformal field theories and supergravity," International Journal of Theoretical Physics, vol. 38, no. 4, pp. 1113-1133, 1999.

[2] J. M. Maldacena, “The Large N Limit of Superconformal Field Theories and Supergravity," Advances in Theoretical and Mathematical Physics, vol. 2, no. 2, pp. 231-252, 1998.

[3] S. S. Gubser, I. R. Klebanov, and A. M. Polyakov, "Gauge theory correlators from non-critical string theory," Physics Letters. B. Particle Physics, Nuclear Physics and Cosmology, vol. 428, no. 12, pp. 105-114, 1998.

[4] E. Witten, "Anti de Sitter space and holography," Advances in Theoretical and Mathematical Physics, vol. 2, no. 2, pp. 253-291, 1998.

[5] T. Banks, L. J. Dixon, D. Friedan, and E. Martinec, "Phenomenology and conformal field theory or can string theory predict the weak mixing angle?" Nuclear Physics B, vol. 299, no. 3, pp. 613-626, 1988. 
[6] V. Balasubramanian, S. B. Giddings, and A. Lawrence, "What do CFTs tell us about Anti-de Sitter space-times?" The Journal of High Energy Physics, vol. 9903, 1999.

[7] I. Bena, "On the construction of local fields in the bulk of AdS(5) and other spaces," Physical Review. D. Third Series, vol. 62, Article ID 066007, 2000.

[8] A. Hamilton, D. Kabat, G. Lifschytz, and D. A. Lowe, "Holographic representation of local bulk operators," Physical Review. D. Third Series, vol. 74, Article ID 066009, 2006.

[9] A. Hamilton, D. Kabat, G. Lifschytz, and D. A. Lowe, "Local bulk operators in AdS/CFT: A Holographic description of the black hole interior," Physical Review D. Particles, Fields, Gravitation, and Cosmology, vol. 75, Article ID 106001, 2007.

[10] A. Hamilton, D. Kabat, G. Lifschytz, and D. A. Lowe, "Publisher's Note: Local bulk operators in AdS/CFT correspondence: A holographic description of the black hole interior," Physical Review D. Particles, Fields, Gravitation, and Cosmology, vol. 75, Article ID 129902, 2007.

[11] L. Susskind and E. Witten, "The Holographic bound in anti-de Sitter space," High Energy Physics, Article ID 9805114, 1998.

[12] I. Heemskerk and J. Polchinski, "Holographic and Wilsonian renormalization groups," Journal of High Energy Physics, vol. 1106, 2011.

[13] D. Sarkar, “(A)dS holography with a cutoff," Physical Review D, vol. 90, no. 8, Article ID 086005, 2014.

[14] V. Balasubramanian, P. Kraus, and A. Lawrence, "Bulk versus boundary dynamics in anti-de Sitter spacetime," Physical Review. D. Third Series, vol. 59, no. 4, 046003, 16 pages, 1999.

[15] D. Z. Freedman, S. D. Mathur, A. Matusis, and L. Rastelli, "Correlation functions in the CFT(d) / AdS $(\mathrm{d}+1)$ correspondence," Nucl. Phys. B, vol. 546, no. 1-2, pp. 96-118, 1999.

[16] D. Pappadopulo, S. Rychkov, J. Espin, and R. Rattazzi, "Operator product expansion convergence in conformal field theory," Physical Review D - Particles, Fields, Gravitation and Cosmology, vol. 86, no. 10, Article ID 105043, 2012.

[17] S. Rychkov, "EPFL Lectures on Conformal Field Theory in D $\geq$ 3 Dimensions," High Energy Physics, 2016.

[18] D. Kabat, G. Lifschytz, and D. A. Lowe, "Constructing local bulk observables in interacting AdS/CFT," Physical Review D - Particles, Fields, Gravitation and Cosmology, vol. 83, no. 10, Article ID 106009, 2011.

[19] Z.-L. Wang, “Talk preseneted on KIAS-YITP Joint Workshop 2015: geometry in gauge theories and string theory".

[20] A. A. Belavin, A. M. Polyakov, and A. B. Zamolodchikov, "Infinite conformal symmetry in two-dimensional quantum field theory," Nuclear Physics. B. Theoretical, Phenomenological, and Experimental High Energy Physics. Quantum Field Theory and Statistical Systems, vol. 241, no. 2, pp. 333-380, 1984.

[21] E. S. Fradkin and M. Y. Palchik, Conformal Quantum Field Theory in D-dimensions, Kluwer Academic Publishers, 1996.

[22] E. S. Fradkin and M. Y. Palchik, "New developments in Ddimensional conformal quantum field theory," Physics Reports. A Review Section of Physics Letters, vol. 300, no. 1-2, 111 pages, 1998.

[23] A. Almheiri, D. Marolf, J. Polchinski, and J. Sully, "Black holes: Complementarity or firewalls?" Journal of High Energy Physics, vol. 2013, no. 2, pp. 1-19, 2013.

[24] S. L. Braunstein, S. Pirandola, and K. Zyczkowski, "Better late than never: Information retrieval from black holes," Physical Review Letters, vol. 110, no. 10, Article ID 101301, 2013.
[25] Y. Nakayama and H. Ooguri, "Bulk locality and boundary creating operators," Journal of High Energy Physics, vol. 2015, no. 10, 4 pages, 2015.

[26] M. Miyaji, T. Numasawa, N. Shiba, T. Takayanagi, and K. Watanabe, "cMERA as Surface/State Correspondence in AdS/CFT," Physical Review Letters, vol. 115, Article ID 171602, 2015.

[27] V. Balasubramanian, P. Kraus, A. Lawrence, and S. P. Trivedi, "Holographic probes of anti-de Sitter spacetimes," Physical Review. D. Third Series, vol. 59, no. 10, Article ID 104021, 14 pages, 1999.

[28] K. Skenderis and B. C. van Rees, "Real-time gauge/gravity duality: prescription, renormalization and examples," Journal of High Energy Physics. A SISSA Journal, vol. 0905, 2009.

[29] D. T. Son and A. O. Starinets, "Minkowski space correlators in AdS / CFT correspondence: Recipe and applications," Journal of High Energy Physics. A SISSA Journal, vol. 0209, 2002.

[30] D. Marolf, "States and boundary terms: Subtleties of Lorentzian AdS / CFT," Journal of High Energy Physics. A SISSA Journal, vol. 0505, 2005.

[31] N. Iqbal and H. Liu, "Real-time response in AdS/CFT with application to spinors," Fortschritte der Physik. Progress of Physics, vol. 57, 2009. 

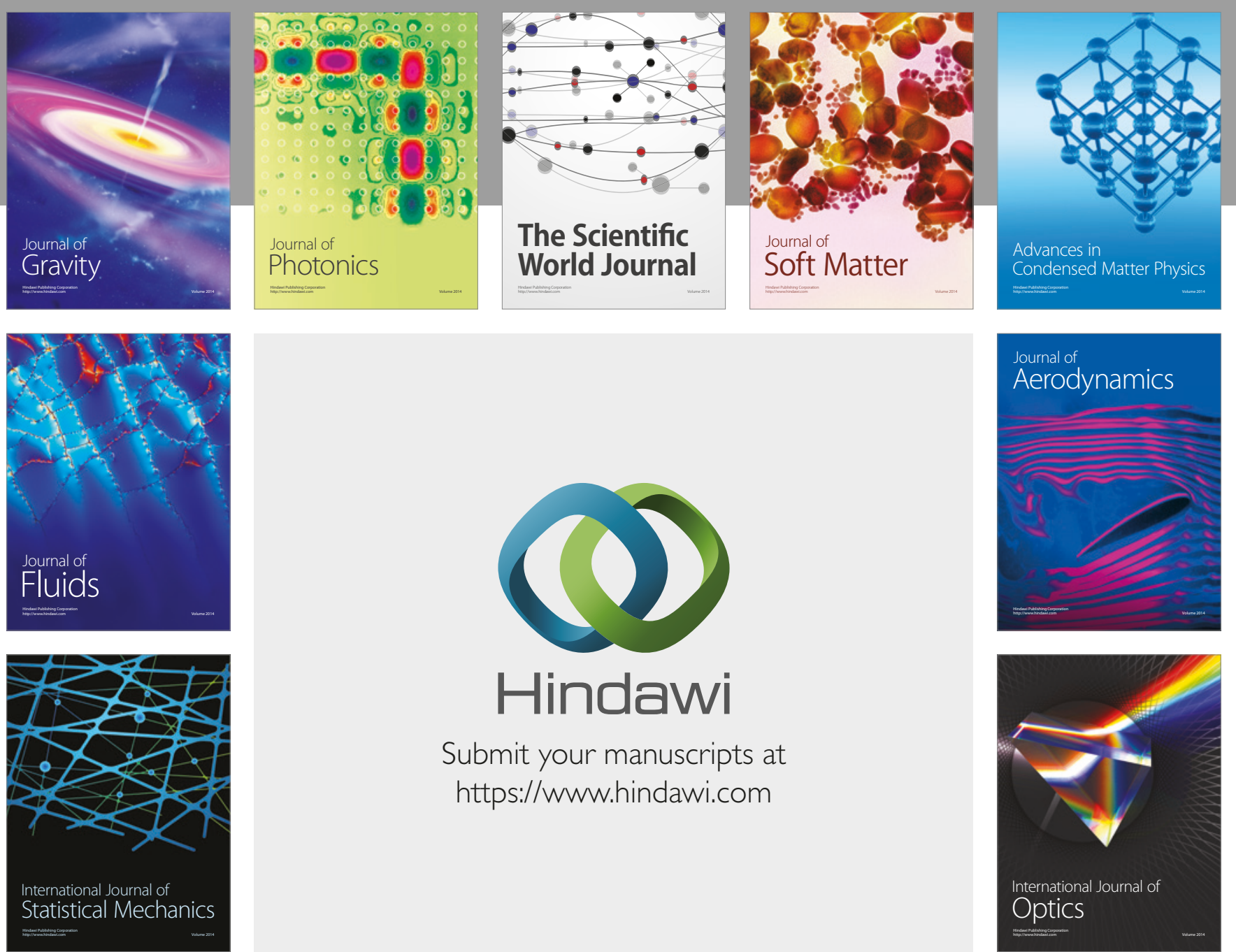

Submit your manuscripts at

https://www.hindawi.com
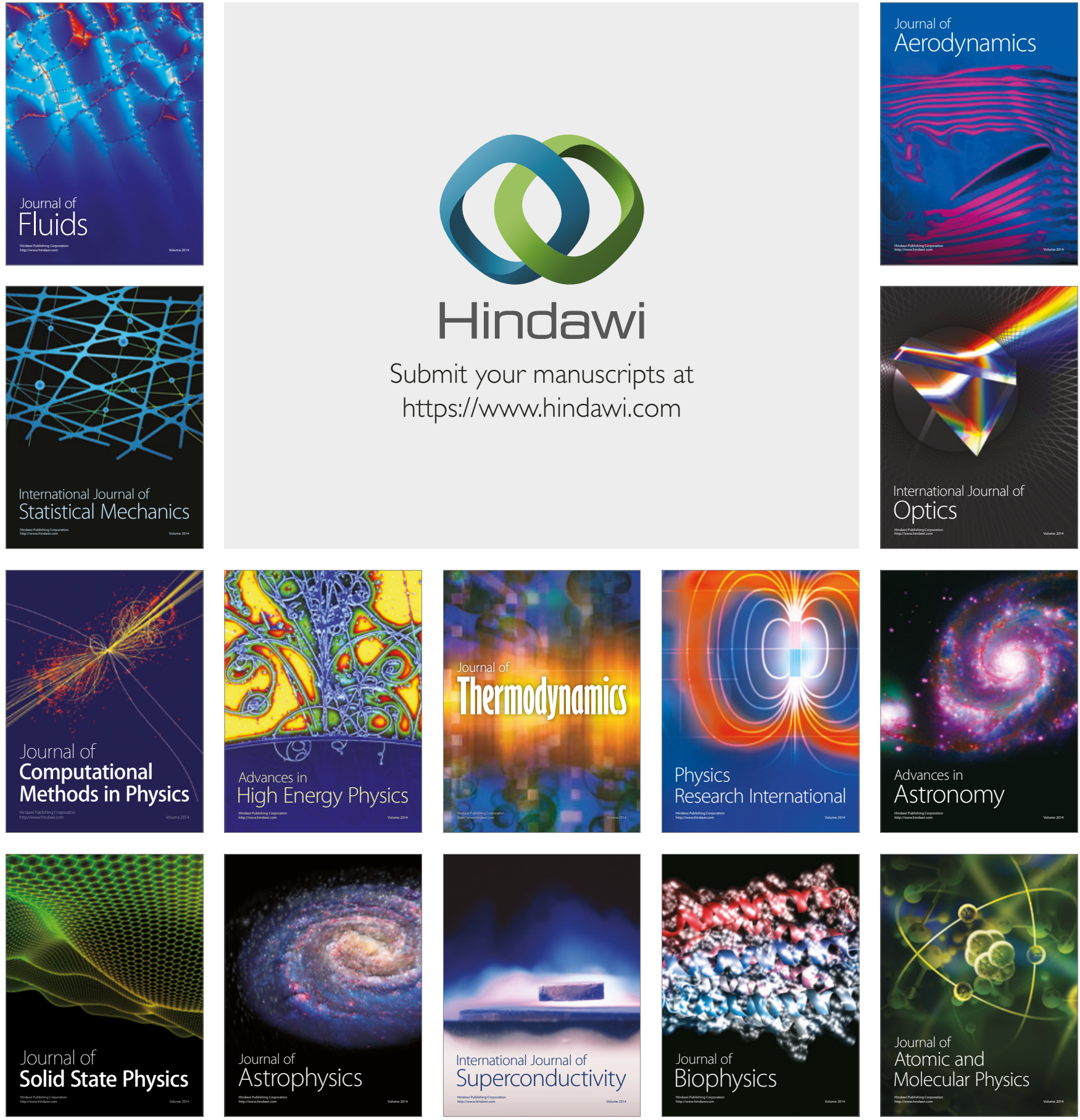